\title{
A checklist of the amphibians and reptiles of San Isidro de Dota, Reserva Forestal Los Santos, Costa Rica
}

\author{
Erick Arias ${ }^{*}$ and Federico Bolaños \\ Universidad de Costa Rica, Escuela de Biología, Museo de Zoología. San Pedro, 11501-2060, San José, Costa Rica. \\ * Corresponding author. E-mail: eapiedra@gmail.com
}

\begin{abstract}
We present an inventory of amphibians and reptiles of San Isidro de Dota, northwest of the Cordillera de Talamanca in the Central Pacific of Costa Rica. The study was conduced from January to August 2012 in premontane wet forest from $689 \mathrm{~m}$ to $800 \mathrm{~m}$ elevation. We found a total of 56 species, including 30 species of amphibians and 26 of reptiles. It results striking the presence of the frog Leptodactylus insularum, uncommon above $400 \mathrm{~m}$ elevation, and the lizard Coloptychon rhombifer, a very uncommon species.
\end{abstract}

\section{INTRODUCTION}

Lower Central America represents one of the regions with the highest number of amphibians described in the Neotropics in relation to the area it represent (Savage 2002; Boza-Oviedo et al. 2012; Hertz et al. 2012). Much of this richness of species is associated with the Cordillera de Talamanca, site of speciation and dispersion center of several species (Streicher et al. 2009; Chaves et al. 2009; Boza-Oviedo et al. 2012). This cordillera extends from the Central Valley of Costa Rica to the southeast and continuing to western Panama. The herpetofauna has been well documented only in very few sites due to the difficult access to many of the sites in this mountain ranges, hence, several sites explored in the past decade have brought out the discovery of many new species of amphibians and reptiles (Poe and Ibañez 2007; Wake et al. 2007; GarcíaParís et al. 2008; Bolaños and Wake 2009; Chaves et al. 2009; Boza-Oviedo et al. 2012).

Biodiversity inventories are needed for population management, conservation and education plans (Caughley and Gunn 1996); however, in the Cordillera de Talamanca these inventories are even more important given the high degree the endemism in groups as amphibians and reptiles where $27 \%$ of the species are endemic to this region (Savage 2002). Also taking into account the decline of several populations of amphibians in Costa Rica (Whitfield et al. 2007), including some localities in this mountain range (Lips 1998), it is relevant to make inventories regarding the composition of amphibians in the region. This list presents the first inventory of amphibians and reptiles of San Isidro de Dota, northwest of the Cordillera de Talamanca in Central Pacific of Costa Rica, a site unexplored scientifically.

\section{MATERIALS AND MethodS}

Study area

The inventory was conducted in the Reserva Forestal Los Santos, in the Central Pacific of Costa Rica (Figure $1)$, in the community of San Isidro de Dota $\left(9^{\circ} 29^{\prime} 31.34^{\prime \prime}\right.$
$\mathrm{N}, 83^{\circ} 58^{\prime} 32.41^{\prime \prime} \mathrm{W}$, WGS84 datum, from $689 \mathrm{~m}$ to 800 $\mathrm{m}$ elevation). The region is dominated by premontane wet forest (Bolaños et al. 1999) with several sites used for agriculture and pastures. The region presents the climate of the pacific slope of the Cordillera de Talamanca, characterized by moderate temperatures and dry periods from January to March, an average annual rainfall of $2190 \mathrm{~mm}$ with an average of 147 rainy days, and a biotemperature that ranges from $17-24^{\circ} \mathrm{C}$ (Solano and Villalobos 2001).

\section{Data collection}

Fieldwork was conducted from January to August 2012, during daily and nightly checks. Total sampling effort was 33 days and 384 hours of active random searches. We sampled the forest, ponds, streams and open areas around human constructions, using the active search and visual encounter survey. According to the distribution ranges given by Savage (2002) and Solórzano (2004) a list of possible species was performed. The individuals collected were euthanized with alcohol, fixed in formalin 10\% and stored in alcohol 70\%. We follow Savage (2002) and Bolaños et al. (2011) for the identification and taxonomy of the specimens. The individuals were collected according to permit granted by SINAC to FB (007-2012-SINAC). Vouchers were deposited in the herpetological collection of the Museo de Zoología, Universidad de Costa Rica (UCR).

\section{RESULTS}

We recorded a total of 56 species (Table 1; Figures 2, 3 and 4), 30 species of amphibians ( 3 orders, 9 families and 20 genera) and 26 species of reptiles ( 1 order, 13 families and 20 genera). These data represent approximately $12.4 \%$ of the herpetofauna of Costa Rica (Bolaños et al. 2011), and $28 \%$ of the herpetofauna of the herpetological province of Montane Slopes and Cordillera Central located on the pacific slope from $600 \mathrm{~m}$ to $1600 \mathrm{~m}$ elevation (Savage 2002). We found $73 \%$ of the species of amphibians proposed and $40 \%$ of reptiles proposed. 


\section{Discussion}

The species richness of the study area was less in comparison with the San Vito region, in the extreme southwestern of the Cordillera de Talamanca in Costa Rica (Santos-Barrera et al. 2008), a site located in the same herpetological province with 67 species of amphibians and reptiles. The difference in species richness found at both sites can be attributable to the effect of altitude on San Vito region, since the sampling sites were located between $1100 \mathrm{~m}$ to $1500 \mathrm{~m}$, but mainly to greater sampling effort, 648 hours, conducted by Santos-Barrera et al. (2008). This effort is reflected in the number of snakes found on the San Vito region, 25 against 10 on San Isidro de Dota, given that the effort should be greater to find snakes by its secretive behavior (Green 1997). Instead, in San Isidro de Dota the amphibian richness was higher in comparison to the San Vito region, 30 against 26.
It was interesting the presence of Leptodactylus insularum to $689 \mathrm{~m}$ elevation, a species uncommon above $400 \mathrm{~m}$ elevation (Savage 2002). San Isidro de Dota is located just $5 \mathrm{~km}$ from the nearest locality of L. insularum $\left(9^{\circ} 27^{\prime} 31.10^{\prime \prime} \mathrm{N},-83^{\circ} 56^{\prime} 38.36^{\prime \prime} \mathrm{W}, 433 \mathrm{~m}\right.$ elevation), however this site is within the altitudinal range considered common for this species. Also highlights the presence of Coloptychon rhombifer which was observed but no specimens were collected, this species is know from only four vouchers in colections (ZMB 8655, UCR $3143,6971,15000$ ) and a photographic voucher (Dwyer and De Plecker 2013). The list of species presented here, mainly with the reptiles, can significantly increase with more effort, however, we consider that this inventory contributes to the knowledge of the herpetofauna of the region and provides a basis for future inventories in this region and surrounding sites.

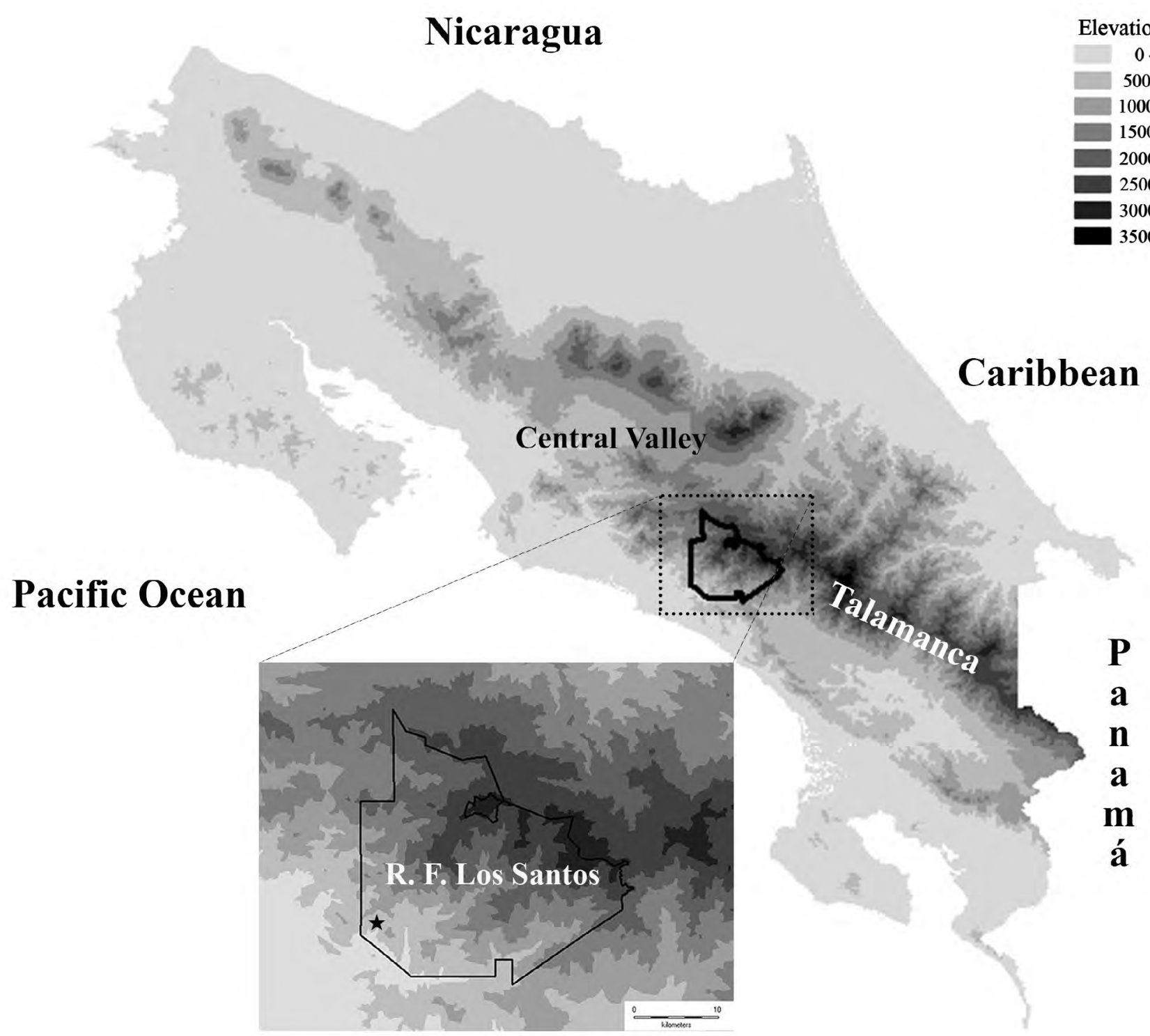

FIGURE 1. Location of study site in the community of San Isidro de Dota (star) in the Reserva Forestal Los Santos, Central Pacific, Costa Rica. 

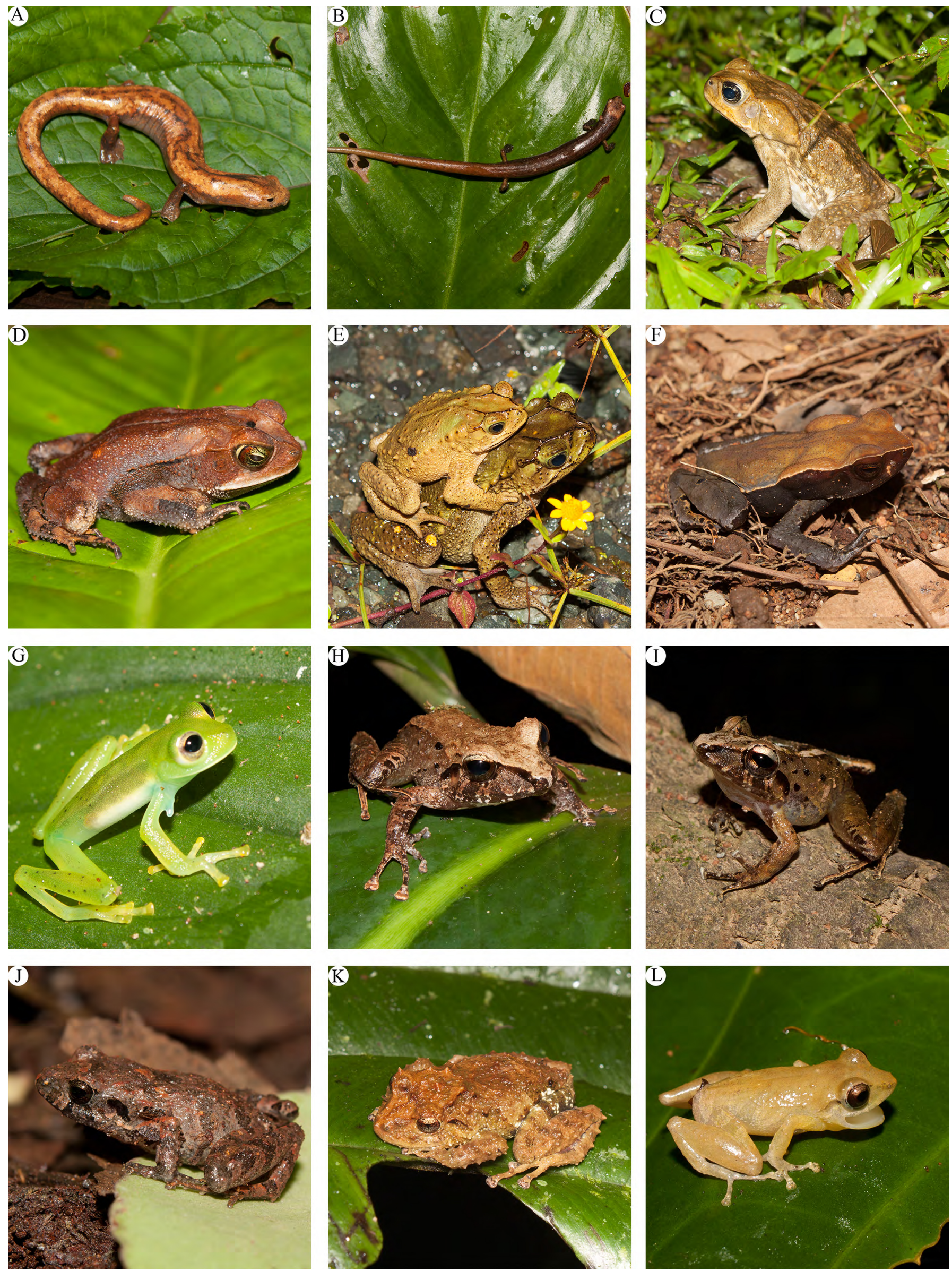

FiguRE 2. Some species of amphibians of San Isidro de Dota, Reserva Forestal Los Santos, Central Pacific, Costa Rica. A) Bolitoglossa lignicolor; B) Oedipina alleni; C) Chaunus marinus; D) Incilius aucoinae; E) Incilius coniferus; F) Rhaebo haematiticus; G) Espadarana prosoblepon; H) Craugastor crassidigitus; I) Craugastor fitzingeri; J) Craugastor stejnegerianus; K) Pristimantis cruentus; L) Diasporus diastema. 

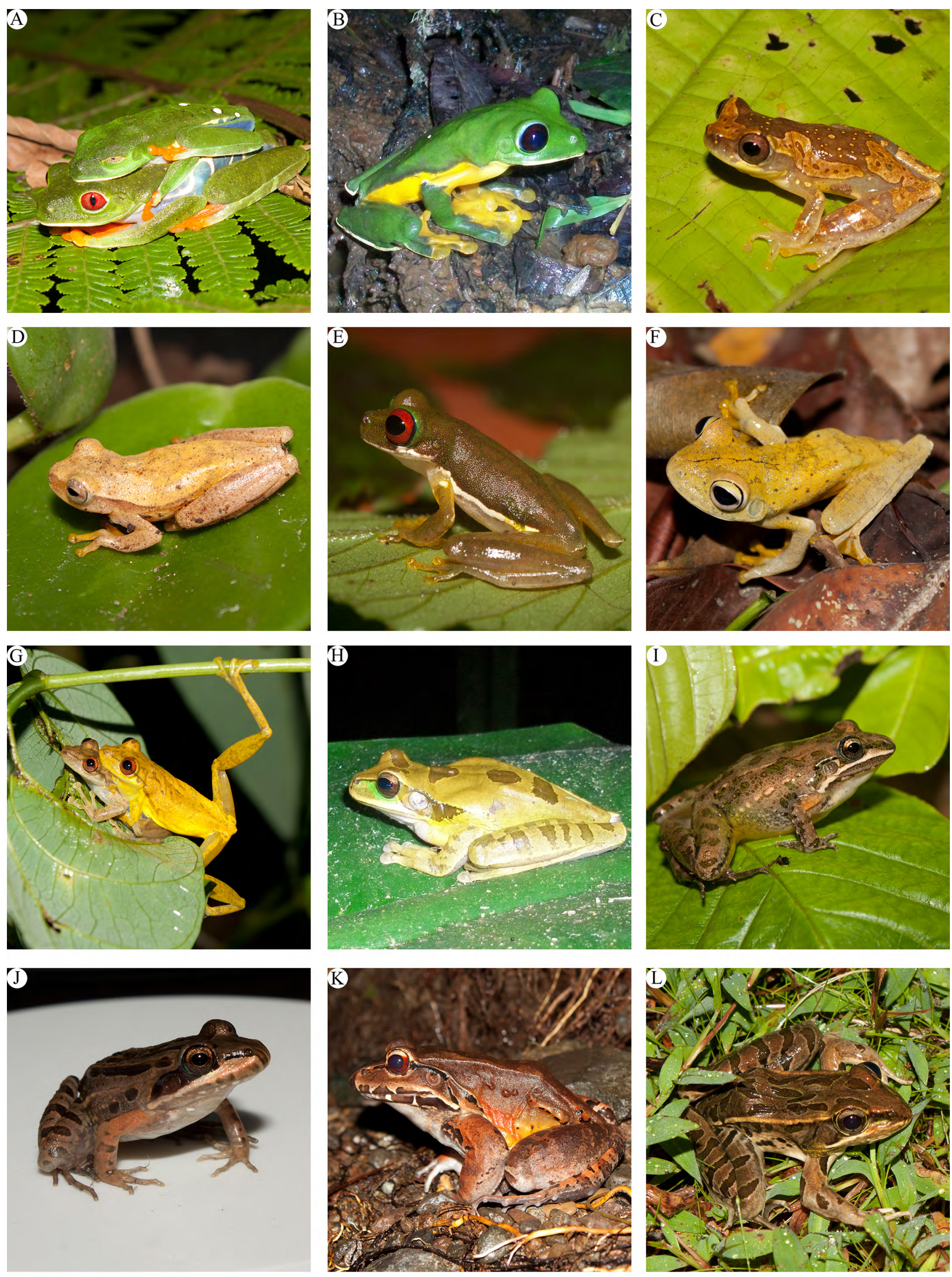

Figure 3. Some species of amphibians of San Isidro de Dota, Reserva Forestal Los Santos, Central Pacific, Costa Rica. A) Agalychnis callidryas; B) Agalychnis spurreli; C) Dendropsophus ebraccatus; D) Dendropsophus microcephalus; E) Duellmanohyla rufioculis; F) Hypsiboas rosenbergi; G) Scinax elaeochroa; H) Smilisca phaeota; I) Leptodactylus fragilis; J) Leptodactylus insularum; K) Leptodactylus savagei; L) Lithobates forreri; 

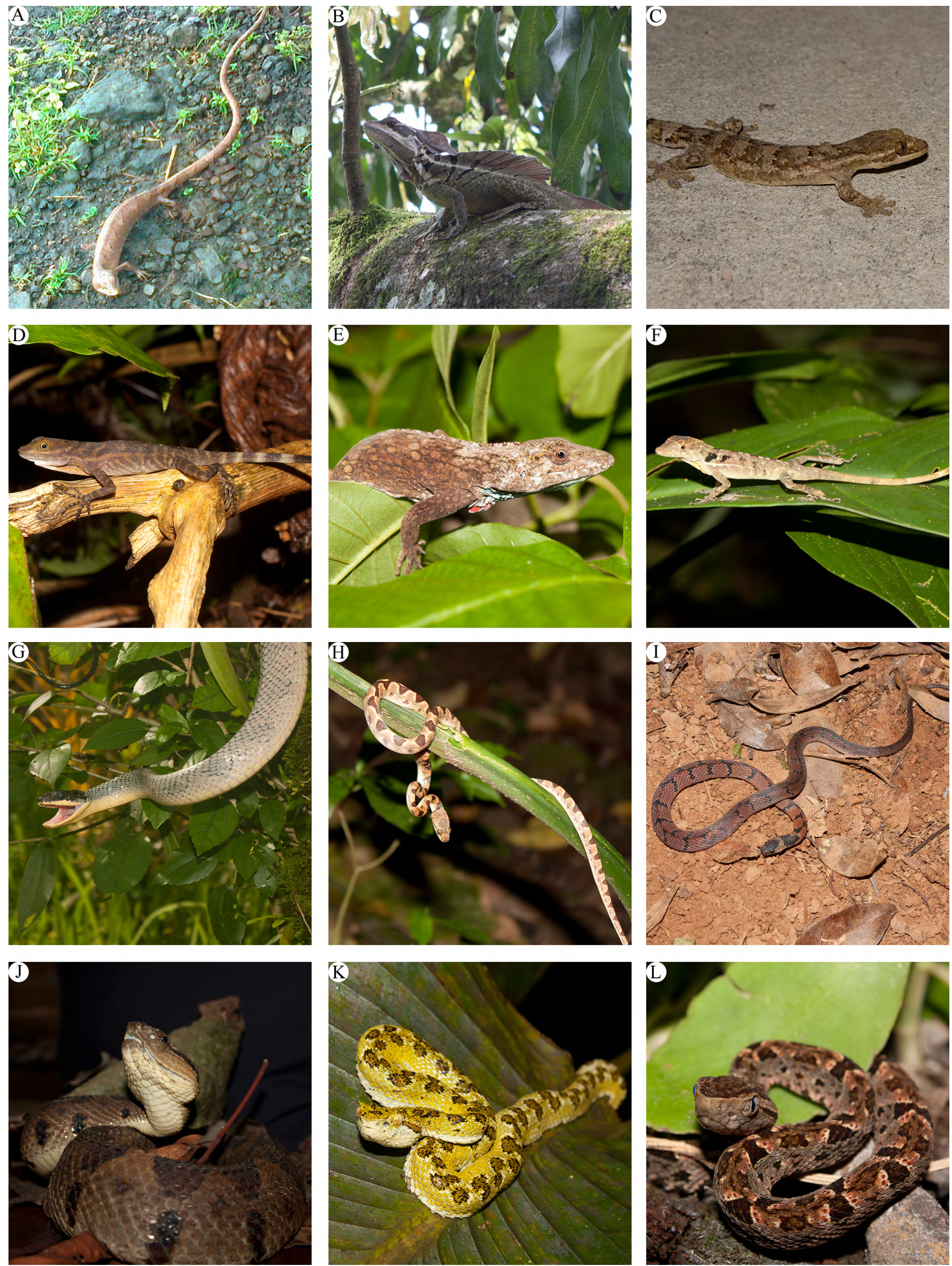

FIGURE 4. Some species of reptiles of San Isidro de Dota, Reserva Forestal Los Santos, Central Pacific, Costa Rica. A) Coloptychon rhombifer (based on a photograph with locality); B) Basiliscus basiliscus; C) Thecadactylus rapicauda; D) Norops aquaticus; E) Norops charlesmyersi; F) Norops polylepis; G) Pseustes poecilonotus; H) Leptodeira septentrionalis; I) Ninia maculata; J) Atropoides mexicanus; K) Bothriechis schlegelii; L) Bothrops asper. 
TABLE 1. Species collected in San Isidro de Dota, Reserva Forestal Los Santos in the Central Pacific of Costa Rica. *Visual Record.

\begin{tabular}{|c|c|c|c|c|}
\hline \multirow{2}{*}{ SPECIES } & \multicolumn{4}{|c|}{ HABITAT } \\
\hline & FOREST & PONDS & STREAMS & OPEN AREAS \\
\hline \multicolumn{5}{|l|}{ Amphibia } \\
\hline \multicolumn{5}{|l|}{ Gymnophiona } \\
\hline \multicolumn{5}{|l|}{ Dermophiidae } \\
\hline Dermophis glandulosus (Taylor, 1955) & & & & $\mathrm{X}$ \\
\hline \multicolumn{5}{|l|}{ Caudata } \\
\hline \multicolumn{5}{|l|}{ Plethodontidae } \\
\hline Bolitoglossa lignicolor (W. Peters, 1873) & & & $\mathrm{X}$ & \\
\hline Oedipina alleni (Taylor, 1954) & $\mathrm{X}$ & & & \\
\hline \multicolumn{5}{|l|}{ Anura } \\
\hline \multicolumn{5}{|l|}{ Bufonidae } \\
\hline Chaunus marinus (Linné, 1758) & & $\mathrm{X}$ & & $\mathrm{X}$ \\
\hline Incilius aucoinae (O’Neill \& Mendelson, 2004) & $\mathrm{X}$ & & & \\
\hline Incilius coniferus (Cope, 1862) & $\mathrm{X}$ & & & $\mathrm{X}$ \\
\hline Rhaebo haematiticus (Cope, 1862) & & & $\mathrm{X}$ & \\
\hline \multicolumn{5}{|l|}{ Centrolenidae } \\
\hline Espadarana prosoblepon (Boettger, 1892) & & & $\mathrm{X}$ & \\
\hline \multicolumn{5}{|l|}{ Craugastoridae } \\
\hline Craugastor crassidigitus (Taylor, 1952) & $\mathrm{X}$ & & & $\mathrm{X}$ \\
\hline Craugastor fitzingeri (0. Schmidt, 1857) & $\mathrm{X}$ & & & $\mathrm{X}$ \\
\hline Craugastor stejnegerianus (Cope, 1893) & $X$ & & & $X$ \\
\hline Pristimantis cruentus (W. Peters, 1873) & $\mathrm{X}$ & & & \\
\hline Pristimantis ridens (Cope, 1866) & $\mathrm{X}$ & & & $\mathrm{X}$ \\
\hline \multicolumn{5}{|l|}{ Eleutherodactylidae } \\
\hline Diasporus diastema (Cope, 1875) & $\mathrm{X}$ & & & $\mathrm{X}$ \\
\hline Diasporus vocator (Taylor, 1955) & $\mathrm{X}$ & & & $\mathrm{X}$ \\
\hline \multicolumn{5}{|l|}{ Hylidae } \\
\hline Agalychnis callidryas (Cope, 1862) & & $\mathrm{X}$ & & $\mathrm{X}$ \\
\hline Agalychnis spurrelli (Boulenger, 1913) & & $\mathrm{X}$ & & \\
\hline Dendropsophus ebraccatus (Cope, 1874) & & $X$ & & \\
\hline Dendropsophus microcephalus (Cope, 1886) & & $X$ & & \\
\hline Duellmanohyla rufioculis (Taylor, 1952) & & & $\mathrm{X}$ & \\
\hline Hypsiboas rosenbergi (Boulenger, 1898) & & & & $\mathrm{X}$ \\
\hline Isthmohyla pseudopuma (Günther, 1901) & & & & $X$ \\
\hline Scinax elaeochroa (Cope, 1875) & & $\mathrm{X}$ & & $\mathrm{X}$ \\
\hline Smilisca phaeota (Cope, 1862) & & $\mathrm{X}$ & & $X$ \\
\hline Smilisca sordida (W. Peters, 1863) & & $\mathrm{X}$ & & \\
\hline Trachycephalus venulosus (Laurenti, 1768) & & & & $\mathrm{X}$ \\
\hline \multicolumn{5}{|l|}{ Leptodactylidae } \\
\hline Leptodactylus fragilis (Brocchi, 1877) & & & & $\mathrm{X}$ \\
\hline Leptodactylus insularum (Barbour, 1906) & & & & $\mathrm{X}$ \\
\hline Leptodactylus savagei (Heyer, 2005) & & $\mathrm{X}$ & $\mathrm{X}$ & $\mathrm{X}$ \\
\hline \multicolumn{5}{|l|}{ Ranidae } \\
\hline Lithobates forreri (Boulenger, 1883) & & $\mathrm{X}$ & & \\
\hline \multicolumn{5}{|l|}{ Reptilia } \\
\hline \multicolumn{5}{|l|}{ Sauria } \\
\hline Anguidae & & & & \\
\hline Coloptychon rhombifer (W. Peters, 1876) & & & & $*$ \\
\hline Diploglossus bilobatus (O’Shaughnessy, 1874) & & & & $\mathrm{X}$ \\
\hline Corytophanidae & & & & \\
\hline Basiliscus basiliscus (Linné, 1758) & & & $\mathrm{X}$ & \\
\hline Gekkonidae & & & & \\
\hline Lepidoblepharis xanthostigma (Noble, 1916) & & & & $\mathrm{X}$ \\
\hline Thecadactylus rapicauda (Houttuyn, 1782) & & & & $\mathrm{X}$ \\
\hline Gymnophthalmidae & & & & \\
\hline Leposoma southi (Ruthven \& Gaige, 1924) & $\mathrm{X}$ & & & \\
\hline Dactyloidae & & & & \\
\hline Norops aquaticus (Taylor, 1956) & & & $\mathrm{X}$ & \\
\hline Norops biporcatus (Wiegmann, 1834) & & $\mathrm{X}$ & & \\
\hline
\end{tabular}


TABLE 1. Continued.

\begin{tabular}{|c|c|c|c|c|}
\hline \multirow{2}{*}{ SPECIES } & \multicolumn{4}{|c|}{ HABITAT } \\
\hline & FOREST & PONDS & STREAMS & OPEN AREAS \\
\hline Norops charlesmyersi (Köhler, 2010) & & & & $\mathrm{X}$ \\
\hline Norops lemurinus (Cope, 1861) & & & & $\mathrm{X}$ \\
\hline Norops limifrons (Cope, 1862) & $\mathrm{X}$ & & & $\mathrm{X}$ \\
\hline Norops polylepis (W. Peters, 1874) & $\mathrm{X}$ & & $\mathrm{X}$ & $\mathrm{X}$ \\
\hline \multicolumn{5}{|l|}{ Scincidae } \\
\hline Scincella cherriei (Cope, 1893) & & & & $\mathrm{X}$ \\
\hline \multicolumn{5}{|l|}{ Teiidae } \\
\hline Ameiva festiva (Lichtenstein \& von Martens, 1856) & & & & $\mathrm{X}$ \\
\hline Ameiva quadrilineata (Hallowell, 1861) & & & & $\mathrm{X}$ \\
\hline \multicolumn{5}{|l|}{ Xantusiidae } \\
\hline Lepidophyma reticulatum (Taylor, 1955) & & & & $\mathrm{X}$ \\
\hline \multicolumn{5}{|l|}{ Serpentes } \\
\hline \multicolumn{5}{|l|}{ Colubridae } \\
\hline Chironius grandisquamis (W. Peters, 1868) & $\mathrm{X}$ & & & \\
\hline Mastigodryas melanolomus (Cope, 1868) & & & & $\mathrm{X}$ \\
\hline Pseustes poecilonotus (Günther, 1858) & $\mathrm{X}$ & & & $\mathrm{x}$ \\
\hline \multicolumn{5}{|l|}{ Didsadidae } \\
\hline Leptodeira septentrionalis (Kennicott, 1859) & $\mathrm{X}$ & & & $\mathrm{X}$ \\
\hline Ninia maculata (W. Peters, 1861) & & & & $\mathrm{X}$ \\
\hline Sibon nebulatus (Linné, 1758) & $\mathrm{X}$ & & & $\mathrm{x}$ \\
\hline \multicolumn{5}{|l|}{ Elapidae } \\
\hline Micrurus alleni (K. Schmidt, 1936) & & & & $\mathrm{X}$ \\
\hline \multicolumn{5}{|l|}{ Viperidae } \\
\hline Atropoides mexicanus (C. Duméril, Bibron \& A. Duméril, 1854) & $\mathrm{X}$ & & & \\
\hline Bothriechis schlegelii (Berthold, 1845) & $\mathrm{x}$ & & & \\
\hline Bothrops asper (Garman, 1884) & $\mathrm{X}$ & & & $\mathrm{X}$ \\
\hline
\end{tabular}

ACKNOWLEDGments: We thank M.Sc. Gerardo Chaves Cordero for helping in identification of some specimens. Special thanks to Francisco Piedra Chinchilla who provided logistical support and to Ezequiel Ramírez for assistance in the field. Financial support was provided by Vicerrectoría de Acción Social, Universidad de Costa Rica through TC-586.

\section{Literature Cited}

Bolaños, F. and D.B. Wake. 2009. Two new species of montane web-footed salamanders (Plethodontidae: Bolitoglossa) from the Costa RicaPanamá border region. Zootaxa 1981: 57-68.

Bolaños, F., J.M. Savage and G. Chaves. 2011. Anfibios y reptiles de Costa Rica. Accessible at http://museo.biologia.ucr.ac.cr/Listas/ LZAPublicaciones.htm. Captured on 20 March 2013.

Bolaños, R., V. Watson and J. Tosi. 1999. Mapa ecológico de Costa Rica (zonas de vida), según el sistema de clasificación de zonas de vida del mundo de L.R. Holdridge, Escala 1:750 000. San José: Centro Científico Tropical.

Boza-Oviedo, E., S.M. Rovito, G. Chaves, A. García-Rodríguez, L.G. Artavia, F. Bolaños and D.B. Wake. 2012. Salamanders from the eastern Cordillera de Talamanca, Costa Rica, with descriptions of five new species (Plethodontidae: Bolitoglossa, Nototriton, and Oedipina) and natural history notes from recent expeditions. Zootaxa 3309: 36-61.

Caughley, G. and A. Gunn. 1996. Conservation biology in theory and practice. Cambridge: Blackwell Science. 459 pp.

Chaves, G., A. García-Rodríguez, A. Mora and A. Leal. 2009. A new species of dink frog (Anura: Eleutherodactylidae: Diasporus) from Cordillera de Talamanca, Costa Rica. Zootaxa 2088: 1-14.

Dwyer, Q. and R. De Plecker. 2013. Geographic distribution: Coloptychon rhombifer (isthmian alligator lizard). Herpetological Review 44(4): 625.

García-París, M., G. Parra-Olea and D.B. Wake. 2008. Description of a new species of the Bolitoglossa subpalmata group (Caudata: Plethodontidae) from Costa Rica. Herpetological Journal 18: 23-31.

Green, H.W. 1997. Snakes: the evolution of mystery in nature. Berkeley, California: University of California Press. 351 pp.

Hertz, A., F. Hauenschild, S. Lotzkat and G. Koehler. 2012. A new golden frog species of the genus Diasporus (Amphibia, Eleutherodactylidae) from the Cordillera Central, western Panama. ZooKeys 196: 23-46 (doi: 10.3897/zookeys.196.2774).

Lips, K.R. 1998. Decline of a tropical montane amphibian fauna. Conservation Biology 12(1): 106-117.
Poe, S. and R. Ibañez. 2007. A new species of Anolis lizard from the Cordillera de Talamanca of Western Panamá. Journal of Herpetology 41(2): 263-270.

Santos-Barrera, G., J. Pacheco, F. Mendoza-Quijano, F. Bolaños, G. Chaves, G.C. Daily, P.R. Ehrlich and G. Ceballos. 2008. Diversity, natural history and conservation of amphibians and reptiles from the San Vito Region, southwestern Costa Rica. Revista de Biología Tropical 56(2): $755-778$.

Savage, J.M. 2002. The amphibians and reptiles of Costa Rica: A herpetofauna between two continents, between two seas. Chicago, Illinois: University of Chicago Press. $934 \mathrm{pp}$.

Solano, J. and R. Villalobos. 2001. Aspectos fisiográficos aplicados a un bosquejo de regionalización geográfico climático de Costa Rica. Tópicos Meteorológicos y Oceanográficos 8: 26-39.

Solórzano, A. 2004. Serpientes de Costa Rica: Distribución, taxonomía $e$ historia natural. Heredia, Costa Rica: Instituto Nacional de Biodiversidad. 792 pp.

Streicher, J.W., A.J. Crawford and C.W. Edwards. 2009. Multilocus molecular phylogenetic analysis of the montane Craugastor podiciferus species complex (Anura: Craugastoridae) in Isthmian Central America. Molecular Phylogenetics and Evolution 53: 620-630 (doi: 10.1016/j. ympev.2009.07.011).

Wake, D.B., J.M. Savage and J. Hanken. 2007. Montane salamanders from the Costa Rica-Panama border region, with descriptions of two new species of Bolitoglossa. Copeia 2007: 556-565.

Whitfield, S.M., K.E. Bell, T. Philippi, M. Sasa, F. Bolaños, G. Chaves, J.M. Savage and M.A. Donnelly. 2007. Amphibian and reptile declines over 35 years at La Selva, Costa Rica. Proceedings of the National Academy of Sciences of the United States of America 104(20): 8352-8356.

RECEIVED: October 2013

ACCEPTED: June 2014

Published online: September 2014

EDITORIAL RESPONSIBILITY: Pedro H. Bernardo

APPENDIX 1. Specimens collected in the study site.

AMPHIBIA. GYMNOPHIONA: Dermophiidae: Dermophis glandulosus (UCR 21734). CAUDATA: Plethodontidae: Bolitoglossa lignicolor (UCR 21758); Oedipina alleni (UCR 20929, 21841). ANURA: Bufonidae: Chaunus marinus (UCR 21481); Incilius aucoinae (UCR 21489, 21737); I. coniferus (UCR 21543, 21738); Rhaebo haematiticus 
(UCR 20920). Centrolenidae: Espadarana prosoblepon (UCR 21474, 21747, 21748). Craugastoridae: Craugastor crassidigitus (UCR 20927, 21739-21741); C. fitzingeri (UCR 21475, 21745, 21761, 21762, 21764, 21802, 21804); C. stejnegerianus (UCR 21456, 21742, 21765, 21766); Pristimantis cruentus (UCR 21541); P. ridens (UCR 21476, 21767). Eleutherodactylidae: Diasporus diastema (UCR 21477, 21803); D. vocator (UCR 21021). Hylidae: Agalychnis callidryas (UCR 20922, 21780-21781); A. spurreli (UCR 21779); Dendropsophus ebraccatus (UCR 21490, 21798); D. microcephalus (UCR 21457, 21796, 21800, 21805, 21807); Duellmanohyla rufioculis (UCR 20926, 21743, 21744, 21746, 21788, 21790); Hypsiboas rosenbergi (UCR 21484); Isthmohyla pseudopuma (UCR 21948); Scinax elaeochroa (UCR 21787, 21789, 21791, 21792); Smilisca phaeota (UCR 21023, 21759, 21763); S. sordida (UCR 21024, 21542, 21760); Trachycephalus venulosus (UCR 21483). Leptodactylidae: Leptodactylus fragilis (UCR 21736); L. insularum (UCR 21801); L. savagei (UCR 21022). Ranidae: Lithobates forreri (UCR 21461, 21482, 21778, 21786).
REPTILIA. SAURIA: Anguidae: Diploglossus bilobatus (UCR 21757). Corytophanidae: Basiliscus basiliscus (UCR 21470). Gekkonidae: Lepidoblepharis xanthostigma (UCR 21769); Thecadactylus rapicauda (UCR 20935, 21749). Gymnophthalmidae: Leposoma southi (UCR 20930). Dactyloidae: Norops aquaticus (UCR 21750, 21751, 21794); N. biporcatus (UCR 21793); N. charlesmyersi (UCR 21756); N. lemurinus (UCR 20932, 21546); N. limifrons (UCR 21479); N. polylepis (UCR 2145821460, 21752-21755, 21768, 21797). Scincidae: Scincella cherriei (UCR 21028). Teiidae: Ameiva festiva (UCR 21487); A. quadrilineata (UCR 21488). Xantusiidae: Lepidophyma reticulatum (UCR 21480). SERPENTES: Colubridae: Chironius grandisquamis (UCR 21770, 21773); Mastigodryas melanolomus (UCR 21486); Pseustes poecilonotus (UCR 21043, 21546, 21771, 21774). Didsadidae: Leptodeira septentrionalis (UCR 21732, 21785); Ninia maculata (UCR 21545, 21730); Sibon nebulatus (UCR 21544, 21731, 21733). Elapidae: Micrurus alleni (UCR 21485). Viperidae: Atropoides mexicanus (UCR 21548); Bothriechis schlegelii (UCR 21549); Bothrops asper (UCR 21772). 